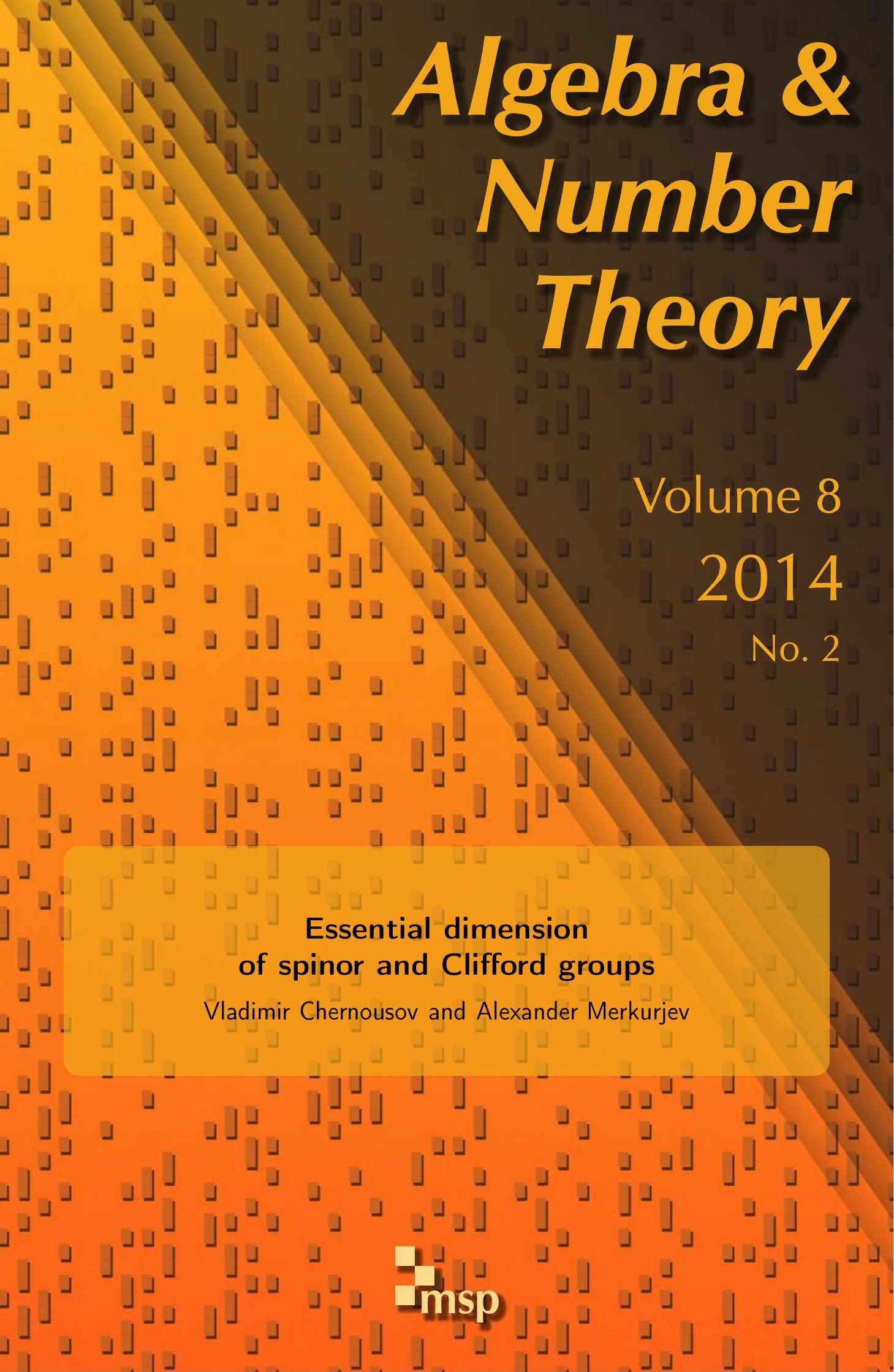




\title{
Essential dimension of spinor and Clifford groups
}

\author{
Vladimir Chernousov and Alexander Merkurjev
}

\begin{abstract}
We conclude the computation of the essential dimension of split spinor groups, and an application to algebraic theory of quadratic forms is given. We also compute essential dimension of the split even Clifford group or, equivalently, of the class of quadratic forms with trivial discriminant and Clifford invariant.
\end{abstract}

\section{Introduction}

We recall briefly the definition of the essential dimension.

Let $F$ be a field, and let $\mathscr{F}:$ Fields $/ F \rightarrow$ Sets be a functor from the category of field extensions over $F$ to the category of sets. Let $E \in$ Fields $F$ and $K \subset E$ a subfield over $F$. We say that $K$ is a field of definition of an element $\alpha \in \mathscr{F}(E)$ if $\alpha$ belongs to the image of the map $\mathscr{F}(K) \rightarrow \mathscr{F}(E)$. The essential dimension of $\alpha$, denoted $\operatorname{ed}^{\mathscr{F}}(\alpha)$, is the least transcendence degree $\operatorname{tr} \cdot \operatorname{deg}_{F}(K)$ over all fields of definition $K$ of $\alpha$. The essential dimension of the functor $\mathscr{F}$ is

$$
\operatorname{ed}(\mathscr{F})=\sup \left\{\operatorname{ed}^{\mathscr{F}}(\alpha)\right\},
$$

where the supremum is taken over all fields $E \in$ Fields $/ F$ and all $\alpha \in \mathscr{F}(E)$ (see [Berhuy and Favi 2003, Definition 1.2] or [Merkurjev 2009, §1]). Informally, the essential dimension of $\mathscr{F}$ is the smallest number of algebraically independent parameters required to define $\mathscr{F}_{F}$ and may be thought of as a measure of complexity of $\mathscr{F}$.

Let $p$ be a prime integer. The essential p-dimension of $\alpha \in \mathscr{F}(E)$, denoted $\operatorname{ed}_{p}^{\mathscr{F}}(\alpha)$, is defined as the minimum of $\operatorname{ed}^{\mathscr{F}}\left(\alpha_{E^{\prime}}\right)$, where $E^{\prime}$ ranges over all finite field extensions of $E$ of degree prime to $p$ and $\alpha_{E^{\prime}}$ is the image of $\alpha$ under the map $\mathscr{F}(E) \rightarrow \mathscr{F}\left(E^{\prime}\right)$. The essential p-dimension of $\mathscr{F}$ is

$$
\operatorname{ed}_{p}(\mathscr{F})=\sup \left\{\operatorname{ed}_{p}^{\mathscr{F}}(\alpha)\right\},
$$

Chernousov's work has been supported in part by the Canada Research Chairs Program and an NSERC research grant. Merkurjev's work has been supported by the NSF grant DMS \#1160206. The authors thank Z. Reichstein for useful comments and suggestions, and the Fields Institute for its hospitality. MSC2010: primary 11E04, 11E57, 11E72; secondary 11E81, 14L35, $20 \mathrm{G} 15$.

Keywords: Linear algebraic groups, spinor groups, essential dimension, torsor, nonabelian cohomology, quadratic forms, Witt rings, the fundamental ideal. 
where the supremum ranges over all fields $E \in$ Fields $/ F$ and all $\alpha \in \mathscr{F}(E)$. By definition, $\operatorname{ed}(\mathscr{F}) \geq \operatorname{ed}_{p}(\mathscr{F})$ for all $p$.

For convenience, we write $\operatorname{ed}_{0}(\mathscr{F})=\operatorname{ed}(\mathscr{F})$, so $\operatorname{ed}_{p}(\mathscr{F})$ is defined for $p=0$ and all prime $p$.

Let $G$ be an algebraic group scheme over $F$. Write $\mathscr{F}_{G}$ for the functor taking a field extension $E / F$ to the set $H_{\text {êt }}^{1}(E, G)$ of isomorphism classes of principal homogeneous $G$-spaces ( $G$-torsors) over $E$. The essential ( $p$-)dimension of $\mathscr{F}_{G}$ is called the essential ( $p$-)dimension of $G$ and is denoted by ed $(G)$ and $\operatorname{ed}_{p}(G)$ (see [Reichstein 2000; Reichstein and Youssin 2000]). Thus, the essential dimension of $G$ measures complexity of the class of principal homogeneous $G$-spaces.

In this paper, we conclude the computation of the essential dimension of the split spinor groups $\mathbf{S p i n}_{n}$ originated in [Brosnan et al. 2010; Garibaldi 2009] and continued in [Merkurjev 2009] (Theorem 2.2). In the missing case $n=4 m \geq 16$, we prove that

$$
\operatorname{ed}_{2}\left(\operatorname{Spin}_{n}\right)=\operatorname{ed}\left(\operatorname{Spin}_{n}\right)=2^{(n-2) / 2}+2^{m}-\frac{n(n-1)}{2}
$$

where $2^{m}$ is the largest power of 2 dividing $n$. The value of ed $\left(\mathbf{S p i n}_{n}\right)$ is surprisingly large. Recall a striking consequence of this (see [Brosnan et al. 2010, Theorem 1-1]): the Pfister number $\operatorname{Pf}(3, n)$ is at least exponential in $n$.

In Theorem 4.2, we give an application in algebraic theory of quadratic forms. Precisely, we determine all pairs $(n, b)$ of natural numbers (with two possible exceptions) such that, for every field $F$, any quadratic form in $I^{3}(F)$ of dimension $n$ contains a subform of trivial discriminant of dimension $b$. This result, stated entirely in terms of algebraic theory of quadratic forms, is proved using the tools of the essential dimension!

Theorem 4.2 is applied later in the paper for the computation of the essential dimension of split even Clifford group $\boldsymbol{\Gamma}_{n}^{+}$or, equivalently, of the functor given by $n$-dimensional quadratic forms with trivial discriminant and Clifford invariant (Theorem 7.1).

We use heavily the work [Popov 1987], where the base field is assumed to be of characteristic zero. This explains the characteristic restriction in most of our results.

\section{Essential dimension of $\operatorname{Spin}_{n}$}

Let $G$ be an algebraic group over $F$, and let $C \subset G$ be a normal subgroup over $F$. For a torsor $E \rightarrow \operatorname{Spec}(F)$ of the group $H:=G / C$, consider the stack $[E / G]$ (see [Vistoli 2005]). Recall that an object of the category $[E / G](K)$ for a field extension $K / F$ is a pair $\left(E^{\prime}, \varphi\right)$, where $E^{\prime}$ is a $G$-torsor over $K$ and $\varphi: E^{\prime} / C \stackrel{\sim}{\rightarrow} E_{K}$ is an isomorphism of $H$-torsors over $K$. The essential dimension ed $[E / G]$ of the 
stack $[E / G]$ is the essential dimension of the functor $K \mapsto$ set of isomorphism classes of objects in $[E / G](K)$.

The following was proven independently by R. Lötscher [2013, Example 3.4]:

Proposition 2.1. Let $C$ be a normal subgroup of an algebraic group $G$ over $F$ and $H=G / C$. Then

$$
\operatorname{ed}(G) \leq \operatorname{ed}(H)+\max \operatorname{ed}[E / G],
$$

where the maximum is taken over all field extensions $L / F$ and all $H$-torsors $E$ over $L$.

Proof. Let $I^{\prime}$ be a $G$-torsor over a field extension $K / F$. Then $I:=I^{\prime} / C$ is an $H$-torsor over $K$. There is a subextension $K_{0} / F$ of $K / F$ and an $H$-torsor $E$ over $K_{0}$ such that there is an isomorphism $\varphi: I \stackrel{\sim}{\rightarrow} E_{K}$ of $H$-torsors and tr.deg $\left(K_{0} / F\right) \leq \operatorname{ed}(H)$.

Consider the stack $[E / G]$ over $K_{0}$. The pair $\left(I^{\prime}, \varphi\right)$ is an object of $[E / G](K)$. There is a subextension $K_{1} / K_{0}$ of $K / K_{0}$ such that $\left(I^{\prime}, \varphi\right)$ is defined over $K_{1}$ and tr.deg $\left(K_{1} / K_{0}\right) \leq \operatorname{ed}[E / G]$. It follows that $I^{\prime}$ is defined over the field $K_{1}$ with

$$
\text { tr.deg }\left(K_{1} / F\right)=\operatorname{tr} \cdot \operatorname{deg}\left(K_{0} / F\right)+\operatorname{tr} \cdot \operatorname{deg}\left(K_{1} / K_{0}\right) \leq \operatorname{ed}(H)+\operatorname{ed}[E / G] .
$$

The following theorem concludes computation of the essential dimension of the spinor groups initiated in [Brosnan et al. 2010; Garibaldi 2009] and continued in [Merkurjev 2009]. We write $\operatorname{Spin}_{n}$ for the split spinor group of a nondegenerate quadratic form of dimension $n$ and maximal Witt index.

If $\operatorname{char}(F) \neq 2$, then the essential dimension of $\operatorname{Spin}_{n}$ has the following values for $n \leq 14$ (see [Garibaldi 2009, §23]):

\begin{tabular}{c|rrrrrrrrr}
$n$ & $\leq 6$ & 7 & 8 & 9 & 10 & 11 & 12 & 13 & 14 \\
\hline $\operatorname{ed}_{2}\left(\operatorname{Spin}_{n}\right)=\operatorname{ed}\left(\mathbf{S p i n}_{n}\right)$ & 0 & 4 & 5 & 5 & 4 & 5 & 6 & 6 & 7
\end{tabular}

In the following theorem, we give the values of $\operatorname{ed}_{p}\left(\operatorname{Spin}_{n}\right)$ for $n \geq 15$ and $p=0$ and 2. Note that $\operatorname{ed}_{p}\left(\mathbf{S p i n}_{n}\right)=0$ if $p \neq 0,2$ as every $\mathbf{S p i n}_{n}$-torsor over a field is split over an extension of degree a power of 2 .

Theorem 2.2. Let $F$ be a field of characteristic zero. For every integer $n \geq 15$, we have

$$
\operatorname{ed}_{2}\left(\mathbf{S p i n}_{n}\right)=\operatorname{ed}\left(\mathbf{S p i n}_{n}\right)= \begin{cases}2^{(n-1) / 2}-n(n-1) / 2 & \text { if } n \text { is odd } \\ 2^{(n-2) / 2}-n(n-1) / 2 & \text { if } n \equiv 2(\bmod 4), \\ 2^{(n-2) / 2}+2^{m}-n(n-1) / 2 & \text { if } n \equiv 0(\bmod 4)\end{cases}
$$

where $2^{m}$ is the largest power of 2 dividing $n$.

Proof. The case $n \geq 15$ and $n$ not divisible by 4 has been considered in [Brosnan et al. 2010, Theorem 3-3]. 
Now assume that $n>15$ and $n$ is divisible by 4 . The inequality " $\geq$ " was obtained in [Merkurjev 2009, Theorem 4.9], so we just need to prove the inequality " $\leq$ ". The case $n=16$ was considered in [Merkurjev 2009, Corollary 4.10]. Assume that $n \geq 20$ and $n$ is divisible by 4 .

Consider the following diagram with exact rows:

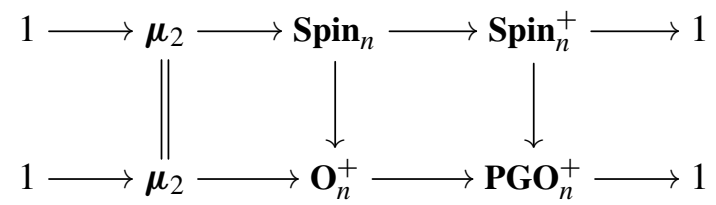

where $\mathbf{S p i n}_{n}^{+}$is the semispinor group, $\mathbf{O}_{n}^{+}$is the split special orthogonal group and $\mathbf{P G O}_{n}^{+}$is the split special projective orthogonal group. We see from the diagram that the image of the connecting map

$$
\delta_{K}: H_{\text {ét }}^{1}\left(K, \operatorname{Spin}_{n}^{+}\right) \rightarrow H_{\text {èt }}^{2}\left(K, \boldsymbol{\mu}_{2}\right) \subset \operatorname{Br}(K)
$$

is contained in the image of the other connecting map

$$
H_{\text {ét }}^{1}\left(K, \mathbf{P G O}_{n}^{+}\right) \rightarrow H_{\text {ét }}^{2}\left(K, \boldsymbol{\mu}_{2}\right) \subset \operatorname{Br}(K)
$$

for every field extension $K / F$. The image of the last map consists of the classes [ $A$ ] of all central simple $K$-algebras $A$ of degree $n$ admitting orthogonal involutions (see [Knus et al. 1998, §31]). As $\operatorname{ind}(A)$ is a power of 2 dividing $n$, we have $\operatorname{ind}(A) \leq 2^{m}$, where $2^{m}$ is the largest power of 2 dividing $n$.

Let $E$ be a $\operatorname{Spin}_{n}^{+}$-torsor over $K$. We have shown that, if $\delta_{K}([E])=[A]$ for a central simple $K$-algebra $A$, then ind $(A) \leq 2^{m}$. It follows from [Brosnan et al. 2011, Theorem 4.1] that $\operatorname{ed}\left[E / \operatorname{Spin}_{n}\right]=\operatorname{ind}(A) \leq 2^{m}$.

It is shown in [Brosnan et al. 2010, Remark 3-10] that

$$
\operatorname{ed}\left(\operatorname{Spin}_{n}^{+}\right)=2^{(n-2) / 2}-\frac{n(n-1)}{2}
$$

for every integer $n \geq 20$ divisible by 4 . Finally, by Proposition 2.1,

$$
\operatorname{ed}\left(\mathbf{S p i n}_{n}\right) \leq \operatorname{ed}\left(\mathbf{S p i n}_{n}^{+}\right)+2^{m}=2^{(n-2) / 2}+2^{m}-\frac{n(n-1)}{2} .
$$

\section{The functors $I_{n}^{k}$}

We use the following notation. Let $F$ be a field of characteristic different from 2 and $K / F$ a field extension. We define

$$
I_{n}^{1}(K)=\begin{aligned}
& \text { Set of isomorphism classes of nondegenerate } \\
& \text { quadratic forms over } K \text { of dimension } n
\end{aligned}
$$


and recall from [Knus et al. 1998, §29.E] the existence of a natural bijection $I_{n}^{1}(K) \simeq H_{\text {ét }}^{1}\left(K, \mathbf{O}_{n}\right)$.

Recall that the discriminant $\operatorname{disc}(q)$ of a form $q \in I_{n}^{1}(K)$ is equal to

$$
(-1)^{n(n-1) / 2} \operatorname{det}(q) \in K^{\times} / K^{\times 2} .
$$

Set

$$
I_{n}^{2}(K)=\left\{q \in I_{n}^{1}(K): \operatorname{disc}(q)=1\right\} .
$$

We have a natural bijection $I_{n}^{2}(K) \simeq H_{\text {êt }}^{1}\left(K, \mathbf{O}_{n}^{+}\right)$(see [Knus et al. 1998, §29.E]).

The Clifford invariant $c(q)$ of a form $q \in I_{n}^{2}(K)$ is the class in the Brauer group $\operatorname{Br}(K)$ of the Clifford algebra of $q$ if $n$ is even and the class of the even Clifford algebra if $n$ is odd [Knus et al. 1998, §8.B]. Define

$$
I_{n}^{3}(K)=\left\{q \in I_{n}^{2}(K): c(q)=0\right\} .
$$

Remark 3.1. Our notation of the functors $I_{n}^{k}$ for $k=1,2,3$ is explained by the following property: $I_{n}^{k}(K)$ consists of all classes of quadratic forms $q \in W(K)$ of dimension $n$ such that $q \in I(K)^{k}$ if $n$ is even and $q \perp\langle-1\rangle \in I(K)^{k}$ if $n$ is odd, where $I(K)$ is the fundamental ideal in the Witt ring $W(K)$ of $K$.

The functor $I_{n}^{3}$ is related to $\mathbf{S p i n}_{n}$-torsors as follows. The short exact sequence

$$
1 \rightarrow \boldsymbol{\mu}_{2} \rightarrow \operatorname{Spin}_{n} \rightarrow \mathbf{O}_{n}^{+} \rightarrow 1
$$

yields an exact sequence

$$
H_{\text {êt }}^{1}\left(K, \boldsymbol{\mu}_{2}\right) \rightarrow H_{\text {êt }}^{1}\left(K, \operatorname{Spin}_{n}\right) \rightarrow H_{\text {êt }}^{1}\left(K, \mathbf{O}_{n}^{+}\right) \stackrel{c}{\rightarrow} H_{\text {êt }}^{2}\left(K, \boldsymbol{\mu}_{2}\right),
$$

where $c$ is the Clifford invariant. Thus, $\operatorname{Ker}(c)=I_{n}^{3}(K)$.

The essential dimensions of $I_{n}^{1}$ and $I_{n}^{2}$ were computed in [Reichstein 2000, Theorems 10.3 and 10.4]: we have ed $\left(I_{n}^{1}\right)=n$ and $\operatorname{ed}\left(I_{n}^{2}\right)=n-1$. In Section 7, we compute ed $\left(I_{n}^{3}\right)$. We will need the following lemma, which was proven in [Brosnan et al. 2010, Lemma 5-1]:

Lemma 3.2. We have $\operatorname{ed}_{p}\left(I_{n}^{3}\right) \leq \operatorname{ed}_{p}\left(\operatorname{Spin}_{n}\right) \leq \operatorname{ed}_{p}\left(I_{n}^{3}\right)+1$ for every $p \geq 0$.

Proof. Let $K / F$ be a field extension. The group $H_{\text {ét }}^{1}\left(K, \mu_{2}\right)=K^{\times} / K^{\times 2}$ acts transitively on the fibers of the second map in the sequence (1). It follows that the natural map $\operatorname{Spin}_{n}$-Torsors $\rightarrow I_{n}^{3}$ is a surjection with $\mathbf{G}_{m}$ acting surjectively on the fibers. The statement follows from [Berhuy and Favi 2003, Proposition 1.13].

Let $\boldsymbol{\Gamma}_{n}^{+}$be the split even Clifford group (see [Knus et al. 1998, §23]). The commutative diagram with exact rows and columns 


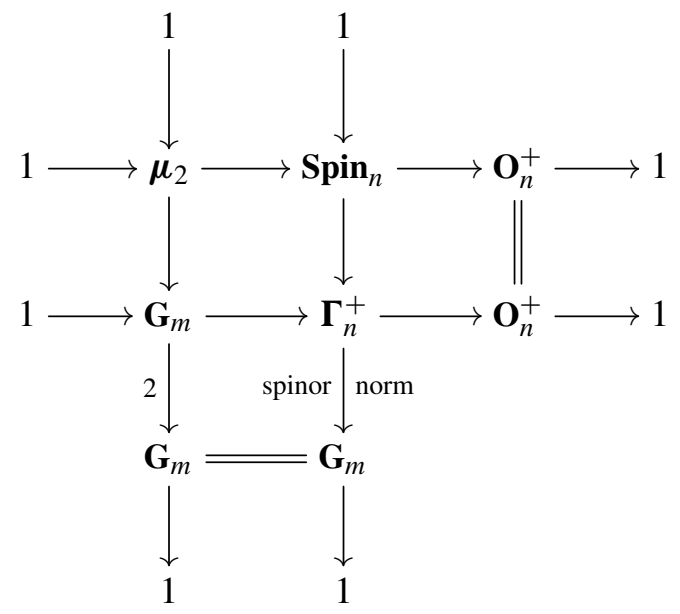

yields a bijection $H_{\text {êt }}^{1}\left(K, \Gamma_{n}^{+}\right) \simeq I_{n}^{3}(K)$ for any field extension $K / F$ (see [Knus et al. 1998, §28]). In particular, $\operatorname{ed}_{p}\left(\boldsymbol{\Gamma}_{n}^{+}\right)=\operatorname{ed}_{p}\left(I_{n}^{3}\right)$.

\section{Subforms of forms in $I_{n}^{3}$}

In this section, we study the following problem in quadratic form theory, which will be used in Section 7 in order to compute the essential dimension of $I_{n}^{3}$. Note that the problem is stated entirely in terms of quadratic forms while in the solution we use the essential dimension. We don't know how to solve the problem by means of quadratic form theory.

Problem 4.1. Given a field $F$, determine all integers $n$ such that every form in $I_{n}^{3}(K)$ contains a nontrivial subform in $I^{2}(K)$ for any field extension $K / F$.

All forms in $I_{n}^{3}(K)$ for $n \leq 14$ are classified (see [Garibaldi 2009, Example 17.8, Theorems 17.13 and 21.3]). Inspection shows that for such $n$ the problem has positive solution.

In the following theorem, we show that in the range $n \geq 15$ the problem has negative solution (with possibly two exceptions):

Theorem 4.2. Let $F$ be a field of characteristic zero, let $n \geq 15$ and let $b$ be an even integer with $0<b<n$. Then there is a field extension $K / F$ and a form in $I_{n}^{3}(K)$ that does not contain a subform in $I_{b}^{2}(K)$ (with possible exceptions $(n, b)=(15,8)$ or $(16,8))$.

Let $a:=n-b$. Write $H_{a, b}$ for the image of the natural homomorphism

$$
\operatorname{Spin}_{a} \times \operatorname{Spin}_{b} \rightarrow \operatorname{Spin}_{n} .
$$

Note that the kernel of (2) is contained in

$$
\boldsymbol{\mu}_{2} \times \boldsymbol{\mu}_{2}=\operatorname{Ker}\left(\operatorname{Spin}_{a} \times \operatorname{Spin}_{b} \rightarrow \mathbf{O}_{a}^{+} \times \mathbf{O}_{b}^{+}\right)
$$


and therefore is the cyclic group of order 2 generated by $(-1,-1)$. Hence, we have an exact sequence

$$
1 \rightarrow \boldsymbol{\mu}_{2} \rightarrow H_{a, b} \rightarrow \mathbf{O}_{a}^{+} \times \mathbf{O}_{b}^{+} \rightarrow 1
$$

and therefore a map

$$
H_{\text {ét }}^{1}\left(R, H_{a, b}\right) \rightarrow H_{\text {ét }}^{1}\left(R, \mathbf{O}_{a}^{+} \times \mathbf{O}_{b}^{+}\right)=H_{\text {ét }}^{1}\left(R, \mathbf{O}_{a}^{+}\right) \times H_{\text {ét }}^{1}\left(R, \mathbf{O}_{b}^{+}\right)
$$

for a commutative $F$-algebra $R$.

We write $q(\eta):=\left(q_{a}, q_{b}\right)$ for the image of an element $\eta \in H_{\text {ét }}^{1}\left(R, H_{a, b}\right)$ under this map, where $q_{a} \in H_{\text {ét }}^{1}\left(R, \mathbf{O}_{a}^{+}\right)$and $q_{b} \in H_{\text {ét }}^{1}\left(R, \mathbf{O}_{b}^{+}\right)$.

Consider the commutative diagram with the exact rows

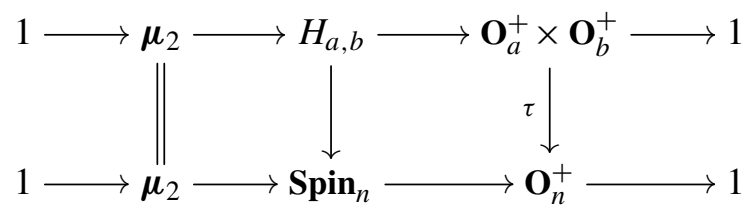

The image of an element $\xi \in H_{\text {ét }}^{1}\left(R, \operatorname{Spin}_{n}\right)$ in $H_{\text {ét }}^{1}\left(R, \mathbf{O}_{n}^{+}\right)$will be denoted by $q(\xi)$.

If $\xi \in H_{\text {ét }}^{1}\left(R, \operatorname{Spin}_{n}\right)$ is the image of an element $\eta \in H_{\text {ét }}^{1}\left(R, H_{a, b}\right)$, then $q(\xi)=$ $q_{a} \perp q_{b}$, the image of $\left(q_{a}, q_{b}\right)=q(\eta)$ under the map induced by $\tau$. We can reverse this statement as follows.

Lemma 4.3. Let $\xi \in H_{e ́ t}^{1}\left(R, \operatorname{Spin}_{n}\right)$ with $q(\xi)=q_{a} \perp q_{b}$, where $q_{a} \in H_{e ́ t}^{1}\left(R, \mathbf{O}_{a}^{+}\right)$ and $q_{b} \in H_{e ̂ t}^{1}\left(R, \mathbf{O}_{b}^{+}\right)$. Then $\xi$ is the image of an element $\eta$ under the map $H_{e ̂ t}^{1}\left(R, H_{a, b}\right) \rightarrow H_{e ̂ t}^{1}\left(R, \operatorname{Spin}_{n}\right)$ such that $q(\eta)=\left(q_{a}, q_{b}\right)$.

Proof. The diagram above yields a commutative diagram with the exact rows

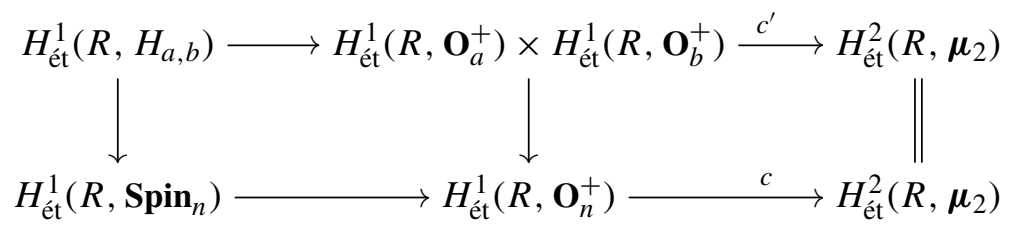

Moreover, the group $H_{\text {ét }}^{1}\left(R, \mu_{2}\right)$ acts transitively on the fibers of the left maps in the two rows. The result follows.

For nonnegative integers $a, b$ and a field extension $K / F$, set

$$
I_{a, b}^{3}(K):=\left\{\left(q_{a}, q_{b}\right) \in I_{a}^{2}(K) \times I_{b}^{2}(K): q_{a} \perp q_{b} \in I_{n}^{3}(K)\right\} .
$$


Corollary 4.4. For any $\eta \in H_{e ̂ t}^{1}\left(K, H_{a, b}\right)$, we have $q(\eta) \in I_{a, b}^{3}(K)$. The morphism of functors $q: H_{a, b}$-Torsors $\rightarrow I_{a, b}^{3}$ is surjective. In particular, $\operatorname{ed}_{p}\left(I_{a, b}^{3}\right) \leq \operatorname{ed}_{p}\left(H_{a, b}\right)$ for every $p \geq 0$.

Proof. Note that the map $c^{\prime}$ in the proof of Lemma 4.3 when $R=K$ takes a pair $\left(q_{a}, q_{b}\right)$ to the Clifford invariant of $q_{a} \perp q_{b}$ in $\operatorname{Br}(K)$. The pair $\left(q_{a}, q_{b}\right) \in$ $I_{a}^{2}(K) \times I_{b}^{2}(K)$ comes from $H_{\text {ét }}^{1}\left(K, H_{a, b}\right)$ if and only if the Clifford invariant of $q_{a} \perp q_{b}$ is split, i.e., $q_{a} \perp q_{b} \in I_{n}^{3}(K)$.

Lemma 4.5. For an even $a$ and any $b$,

$$
\operatorname{ed}_{p}\left(I_{a, b}^{3}\right) \leq \operatorname{ed}_{p}\left(I_{a-1, b}^{3}\right)+1
$$

for every $p \geq 0$.

Proof. Consider the morphism of functors

$$
\alpha: \mathbf{G}_{m} \times I_{a-1, b}^{3} \rightarrow I_{a, b}^{3}, \quad(\lambda ; f, g) \mapsto(\lambda(f \perp\langle-1\rangle), g) .
$$

Every form $h$ in $I_{a}^{2}(K)$ can be written in the form $h=\lambda(f \perp\langle-1\rangle)$ for a value $\lambda$ of $h$ and a form $f \in I_{a-1}^{2}(K)$; i.e., $\alpha$ is a surjection, whence the result.

Write $V_{n}$ and $W_{n}$ for the (semi)spinor and regular representations, respectively, of the group $\operatorname{Spin}_{n}$. We have

$$
\operatorname{dim}\left(V_{n}\right)= \begin{cases}2^{(n-1) / 2} & \text { if } n \text { is odd, } \\ 2^{(n-2) / 2} & \text { if } n \text { is even }\end{cases}
$$

and $\operatorname{dim}\left(W_{n}\right)=n$. We consider the tensor product $V_{a, b}:=V_{a} \otimes V_{b}$ as the representation of the group $H_{a, b}$. We also view $W_{a}$ and $W_{b}$ as $H_{a, b}$-representations via the natural homomorphisms $H_{a, b} \rightarrow \mathbf{O}_{a}^{+}$and $H_{a, b} \rightarrow \mathbf{O}_{b}^{+}$, respectively.

A representation $V$ of an algebraic group $H$ is generically free if the stabilizer of a generic vector in $V$ is trivial. In this case, by [Reichstein and Youssin 2000],

$$
\operatorname{ed}(H) \leq \operatorname{dim}(V)-\operatorname{dim}(H) .
$$

Lemma 4.6. Let $a$ be odd and $b$ even. Suppose that $V_{a, b}$ is a generically free representation of the image of the homomorphism $H_{a, b} \rightarrow \mathbf{G L}\left(V_{a, b}\right)$. Then $V_{a, b} \oplus W_{b}$ is a generically free representation of $H_{a, b}$. In particular,

$$
\operatorname{ed}\left(H_{a, b}\right) \leq \operatorname{dim}\left(V_{a, b}\right)+\operatorname{dim}\left(W_{b}\right)-\operatorname{dim}\left(H_{a, b}\right) .
$$

Proof. Write $C_{n}$ for the kernel of $\mathbf{S p i n}_{n} \rightarrow \mathbf{P G O}_{n}^{+}$and $C_{n}^{\prime}$ for the kernel of $\mathbf{S p i n}_{n} \rightarrow$ $\mathbf{O}_{n}^{+}$, so $C_{n}^{\prime}=\{ \pm 1\} \subset C_{n}$. By assumption, the generic stabilizer $H$ of the action of $\operatorname{Spin}_{a} \times \operatorname{Spin}_{b}$ on $V_{a, b}$ is contained in the center $C_{a} \times C_{b}$. Since $C_{b} / C_{b}^{\prime}=\boldsymbol{\mu}_{2}$ acts on $W_{b}$ by multiplication by -1 , we have $H \subset C_{a} \times C_{b}^{\prime} \simeq \boldsymbol{\mu}_{2} \times \boldsymbol{\mu}_{2}$. Note that $\boldsymbol{\mu}_{2} \times 1$ and $1 \times \mu_{2}$ act by multiplication by -1 on $V_{a, b}$; hence, $H$ is generated by $(-1,-1)$. It follows that $H_{a, b}=\left(\mathbf{S p i n}_{a} \times \mathbf{S p i n}_{b}\right) / H$ acts generically freely on $V_{a, b} \oplus W_{b}$. 
Proposition 4.7. Let $\operatorname{char}(F)=0$. If $n=a+b \geq 15$ with $a \leq b$, then $V_{a, b}$ is a generically free representation of the image of $H_{a, b} \rightarrow \mathbf{G L}\left(V_{a, b}\right)$ if and only if $(a, b) \neq(3,12),(4,11),(4,12),(6,10)$ and $(8,8)$.

Proof. All the cases of infinite generic stabilizers $H$ are listed in [Èlašvili 1972, §3, Row 7 of Table 6]: $H$ is infinite if and only if $(a, b)=(3,12)$ and $(4,12)$.

If $H$ is finite, by [Popov 1987, Theorem 1, Rows 1,12 and 13 of Table 1], $H$ is nontrivial if and only if $(a, b)=(4,11),(6,10)$ and $(8,8)$.

Proof of Theorem 4.2. Note that the case $(n, b)$ with $n$ even implies the case $(n-1, b)$. Indeed, suppose that every form in $I_{n-1}^{3}$ for an even $n$ contains a subform from $I_{b}^{2}$. Take any form $q \in I_{n}^{3}(K)$ for a field extension $K / F$, and write $q=\lambda(f \perp\langle-1\rangle)$ for a $\lambda \in K^{\times}$and $f \in I_{n-1}^{3}(K)$. If $f$ contains a subform $h \in I_{b}^{2}(K)$, then $q$ contains $\lambda h$.

We need to show that the natural morphism of functors $I_{a, b}^{3} \rightarrow I_{n}^{3}$ is not surjective. It suffices to prove that $\operatorname{ed}\left(I_{a, b}^{3}\right)<\operatorname{ed}\left(I_{n}^{3}\right)$. We may assume that $n$ (and hence also $a$ ) is even. Moreover, we may assume that $a \leq b$.

Suppose that $n \geq 18$. By Proposition 4.7, Lemmas 4.5 and 4.6 and Corollary 4.4,

$$
\begin{aligned}
\operatorname{ed}\left(I_{a, b}^{3}\right) & \leq \operatorname{ed}\left(I_{a-1, b}^{3}\right)+1 \\
& \leq \operatorname{ed}\left(H_{a-1, b}\right)+1 \\
& \leq \operatorname{dim}\left(V_{a-1, b}\right)+\operatorname{dim}\left(W_{b}\right)-\operatorname{dim}\left(H_{a-1, b}\right)+1 \\
& =2^{n / 2-2}+b-(a-1)(a-2) / 2-b(b-1) / 2+1 \\
& =2^{n / 2-2}-\left(a^{2}+b^{2}-3 a-3 b\right) / 2 \\
& \leq 2^{n / 2-2}-\left(n^{2}-6 n\right) / 4
\end{aligned}
$$

as $a^{2}+b^{2} \geq n^{2} / 2$. The last integer is strictly less than

$$
2^{n / 2-1}-n(n-1) / 2-1 \leq \operatorname{ed}\left(\operatorname{Spin}_{n}\right)-1 \leq \operatorname{ed}\left(I_{n}^{3}\right)
$$

by Theorem 2.2 and Lemma 3.2.

It remains to consider the case $n=16$. Note that, by Theorem 2.2 and Lemma 3.2,

$$
\operatorname{ed}\left(I_{16}^{3}\right) \geq \operatorname{ed}\left(\operatorname{Spin}_{16}\right)-1=23 .
$$

We shall prove that ed $\left(I_{a, b}^{3}\right)<23$. All possible values of $b$ are 8, 10, 12 and 14 .

Case $(\boldsymbol{n}, \boldsymbol{b})=(\mathbf{1 6}, \mathbf{1 0})$. Consider the representation $V:=W_{6} \oplus V_{6,10} \oplus W_{10}$ of $H_{6,10}$. We claim that $V$ is generically free. The stabilizer in $\mathbf{S p i n}_{6}$ of a point in general position in $W_{6}$ is $\mathbf{S p i n}_{5}$. Hence, the stabilizer in $H_{6,10}$ of a point in general position in $W_{6}$ is $H_{5,10}$. Note that the restriction of $V_{6,10}$ to $H_{5,10}$ is isomorphic to $V_{5,10}$. Finally, the $H_{5,10}$-representation $V_{5,10} \oplus W_{10}$ is generically free by Proposition 4.7. 
It follows from (3) and Corollary 4.4 that

$$
\operatorname{ed}\left(I_{6,10}^{3}\right) \leq \operatorname{ed}\left(H_{6,10}\right) \leq \operatorname{dim}(V)-\operatorname{dim}\left(H_{6,10}\right)=80-60=20 .
$$

Case $(\boldsymbol{n}, \boldsymbol{b})=(\mathbf{1 6}, \mathbf{1 2})$. Consider the representation $V:=W_{3} \oplus W_{3} \oplus V_{3,12} \oplus W_{12}$ of $H_{3,12}$. We claim that $V$ is generically free as the representation of $H_{3,12}$. Indeed, the stabilizer in $H_{3,12}$ of a generic vector in $W_{12}$ is $H_{3,11}$. We are reduced to showing that $W_{3} \oplus W_{3} \oplus V_{3,11}$ is a generically free representation of $H_{3,11}$. By [Popov 1987, $\S 5$, p. 246], the generic stabilizer $S$ of $H_{3,11}$ in $V_{3,11}$ is finite (isomorphic to $\boldsymbol{\mu}_{2} \times \boldsymbol{\mu}_{2}$ ), and the restriction to $S$ of the natural projection $H_{3,11} \rightarrow \mathbf{O}_{3}^{+}$is injective. It remains to notice that the representation $W_{3} \oplus W_{3}$ of $\mathbf{O}_{3}^{+}=\mathbf{P G L}_{2}$ is generically free.

It follows from Lemmas 4.5 and 4.6 and Corollary 4.4 that

$$
\begin{aligned}
\operatorname{ed}\left(I_{4,12}^{3}\right) \leq \operatorname{ed}\left(I_{3,12}^{3}\right)+1 & \leq \operatorname{ed}\left(H_{3,12}\right)+1 \\
& \leq \operatorname{dim}(V)-\operatorname{dim}\left(H_{3,12}\right)+1=82-69+1=14 .
\end{aligned}
$$

Case $(\boldsymbol{n}, \boldsymbol{b})=(\mathbf{1 6}, \mathbf{1 4})$. As every form in $I_{2}^{3}$ is hyperbolic, we have $I_{2,14}^{3}=I_{14}^{3}$ and $\operatorname{ed}\left(I_{14}^{3}\right)=7$ by Theorem 2.2 .

\section{Unramified principal homogeneous spaces}

Let $G$ be an algebraic group over $F$, and let $K / F$ be a field extension with a discrete valuation $v$ trivial on $F$. Write $O$ for the valuation ring of $v$. It is a local $F$-algebra. We say that a class $\xi \in H_{\text {êt }}^{1}(K, G)$ is unramified (with respect to $v$ ) if $\xi$ belongs to the image of the map $H_{\text {ét }}^{1}(O, G) \rightarrow H_{\text {êt }}^{1}(K, G)$.

Let $\bar{K}$ be the residue field of $v$. The ring homomorphism $O \rightarrow \bar{K}$ yields a map $H_{\text {ét }}^{1}(O, G) \rightarrow H_{\text {ét }}^{1}(\bar{K}, G)$. This map is a bijection if $K$ is complete (see [SGA 3 1970, Exposé XXIV, Proposition 8.1]). Hence, we have the map

$$
H_{\text {ét }}^{1}(\bar{K}, G) \stackrel{\sim}{\rightarrow} H_{\text {ét }}^{1}(O, G) \rightarrow H_{\text {ét }}^{1}(K, G)
$$

Example 5.1. Let $\operatorname{char}(F) \neq 2$ and $G=\mathbf{O}_{n}$. Then $H_{\text {êt }}^{1}(K, G)$ is the set of isomorphism classes of nondegenerate quadratic forms of dimension $n$ over $K$. A quadratic form $q$ over a field $K$ with a discrete valuation is unramified if and only if $q \simeq\left\langle a_{1}, a_{2}, \ldots, a_{n}\right\rangle$, where $a_{i}$ are units in the valuation ring $O$ in $K$. In general, every $q$ can be written $q=q_{1} \perp \pi q_{2} \perp h$, where $\pi$ is a prime element, $q_{1}$ and $q_{2}$ are unramified anisotropic quadratic forms and $h$ is a hyperbolic form. The form $q$ is unramified if and only if $q_{2}=0$. It follows that, if two forms $q$ and $\pi q$ are both unramified, then $q$ is hyperbolic. If $K$ is complete, then the map (4) takes $f=\left\langle\bar{a}_{1}, \bar{a}_{2}, \ldots, \bar{a}_{n}\right\rangle$ over $\bar{K}$, where $a_{i}$ are units in $O$, to $f_{K}:=\left\langle a_{1}, a_{2}, \ldots, a_{n}\right\rangle$. 


\section{Essential dimension of $P I_{n}^{3}$}

Two quadratic forms $f$ and $g$ over a field $K$ are called similar if $f=\lambda g$ for some $\lambda \in K^{\times}$. If $n$ is even, we write $P I_{n}^{3}(K)$ for the set of similarity classes of forms in $I_{n}^{3}(K)$. The group $K^{\times}$acts transitively on the fibers of the natural surjective map $I_{n}^{3}(K) \rightarrow P I_{n}^{3}(K)$. Hence,

$$
\operatorname{ed}_{p}\left(P I_{n}^{3}\right) \leq \operatorname{ed}_{p}\left(I_{n}^{3}\right) \leq \operatorname{ed}_{p}\left(P I_{n}^{3}\right)+1
$$

for any $p \geq 0$ by [Berhuy and Favi 2003, Proposition 1.13].

Proposition 6.1. Let $\operatorname{char}(F) \neq 2$. For an even $n \geq 8$, and $p=0$ or 2 , we have

$$
\operatorname{ed}_{p}\left(P I_{n}^{3}\right)=\operatorname{ed}_{p}\left(I_{n}^{3}\right)-1 .
$$

Proof. Let $K / F$ be a field extension, and let $q \in I_{n}^{3}(K)$ be a nonhyperbolic form. Consider the form $t q$ over the field $K((t))$. It suffices to show that

$$
\operatorname{ed}_{p}^{I_{n}^{3}}(t q) \geq \operatorname{ed}_{p}^{P I_{n}^{3}}(q)+1
$$

Let $M / K((t))$ be a finite field extension of degree prime to $p$ (i.e., $M=K((t))$ if $p=0$ and $[M: K((t))]$ is odd if $p=2)$, let $L / F$ be a subextension of $M / F$ and let $f \in I_{n}^{3}(L)$ be such that $\operatorname{tr} \cdot \operatorname{deg}(L / F)=\operatorname{ed}_{p}^{I_{n}^{3}}(t q)$ and $t q_{M} \simeq f_{M}$.

Let $v$ be the (unique) extension on $M$ of the discrete valuation of $K((t))$, and let $w$ be the restriction of $v$ on $L$. The residue field $\bar{M}$ is a finite extension of $K$ of degree prime to $p$. As the form $q$ is not hyperbolic, $q_{M}$ is not hyperbolic, and therefore, the form $t q_{M} \simeq f_{M}$ is ramified by Example 5.1. It follows that $w$ is nontrivial, i.e., $w$ is a discrete valuation on $L$.

Let $\hat{L}$ be the completion of $L$. Note that, as $M$ is complete, we can identify $\hat{L}$ with a subfield of $M$. Write $f_{\hat{L}} \simeq\left(f_{1}\right)_{\hat{L}} \perp \pi\left(f_{2}\right)_{\hat{L}}$, where $f_{1}$ and $f_{2}$ are quadratic forms over the residue field $\bar{L}$ and $\pi \in L$ is a prime element (see Example 5.1). Note that $f_{1}, f_{2} \in I^{2}(\bar{L})$ by [Elman et al. 2008, Lemma 19.4]. If the ramification index $e$ of $M / L$ is even, then $\pi$ is a unit in the valuation ring $O$ of $M$ modulo squares in $M^{\times}$; hence, $f_{M}$ is unramified, a contradiction. It follows that $e$ is odd. Writing $\pi=u t^{e}$ with a unit $u \in O^{\times}$, we have

$$
t q_{M} \simeq f_{M} \simeq\left(f_{1}\right)_{M} \perp \pi\left(f_{2}\right)_{M} \simeq\left(f_{1}\right)_{M} \perp u t\left(f_{2}\right)_{M} ;
$$

hence, $\left(f_{1}\right)_{M}=0$ and $q_{M}=u\left(f_{2}\right)_{M}$ in $W(M)$. It follows that $\left(f_{1}\right)_{\bar{M}}=0$ and $q_{\bar{M}}=\bar{u}\left(f_{2}\right)_{\bar{M}}$ in $W(\bar{M})$, and therefore,

$$
q_{\bar{M}}=\bar{u}\left(f_{2}\right)_{\bar{M}}=\bar{u} g_{\bar{M}},
$$

where $g:=f_{1} \perp f_{2}$ is the form over $\bar{L}$ of dimension $n$. Note that $f_{\hat{L}}-g_{\hat{L}}=$ $\langle\pi,-1\rangle\left(f_{2}\right)_{\widehat{L}} \in I^{3}(\hat{L})$; hence, $g_{\hat{L}} \in I^{3}(\hat{L})$ and $g \in I^{3}(\bar{L})$. 
It follows from (5) that $q_{\bar{M}}$ is similar to $g_{\bar{M}}$, i.e., the form $q$ is $p$-defined over $\bar{L}$ for the functor $P I_{n}^{3}$ (see [Merkurjev 2009, §1.1]), and therefore,

$$
\operatorname{ed}_{p}^{I_{n}^{3}}(t q)=\operatorname{tr} \cdot \operatorname{deg}(L / F) \geq \operatorname{tr} \cdot \operatorname{deg}(\bar{L} / F)+1 \geq \operatorname{ed}_{p}^{P I_{n}^{3}}(q)+1 .
$$

\section{Essential dimension of $\Gamma_{n}^{+}$}

In this section, we compute the essential dimension of $\Gamma_{n}^{+}$and $I_{n}^{3}$.

Theorem 7.1. Let $F$ be a field of characteristic zero. Then for every integer $n \geq 15$ and $p=0$ or 2 , we have

$$
\operatorname{ed}_{p}\left(\boldsymbol{\Gamma}_{n}^{+}\right)=\operatorname{ed}_{p}\left(I_{n}^{3}\right)= \begin{cases}2^{(n-1) / 2}-1-n(n-1) / 2 & \text { if } n \text { is odd } \\ 2^{(n-2) / 2}-n(n-1) / 2 & \text { if } n \equiv 2(\bmod 4) \\ 2^{(n-2) / 2}+2^{m}-1-n(n-1) / 2 & \text { if } n \equiv 0(\bmod 4)\end{cases}
$$

where $2^{m}$ is the largest power of 2 dividing $n$.

If $\operatorname{char}(F) \neq 2$, then the essential dimension of $I_{n}^{3}$ has the following values for $n \leq 14$ :

\begin{tabular}{c|rrrrrrrrr}
$n$ & $\leq 6$ & 7 & 8 & 9 & 10 & 11 & 12 & 13 & 14 \\
\hline $\operatorname{ed}_{2}\left(I_{n}^{3}\right)=\operatorname{ed}\left(I_{n}^{3}\right)$ & 0 & 3 & 4 & 4 & 4 & 5 & 6 & 6 & 7
\end{tabular}

Proof. We will prove the theorem case by case.

Case $n \equiv 2(\bmod 4)$ and $n \geq 10$. The exact sequence

$$
1 \rightarrow \boldsymbol{\mu}_{4} \rightarrow \operatorname{Spin}_{n} \rightarrow \mathbf{P G O}_{n}^{+} \rightarrow 1
$$

yields a surjective map $\operatorname{Spin}_{n}$-Torsors $(K) \rightarrow P I_{n}^{3}(K)$ for any $K / F$, with the group $K^{\times}$acting transitively on the fibers of this map. It follows from Theorem 2.2, Proposition 6.1 and Lemma 3.2 that

$$
\operatorname{ed}_{2}\left(I_{n}^{3}\right)=\operatorname{ed}_{2}\left(P I_{n}^{3}\right)+1 \geq \operatorname{ed}_{2}\left(\operatorname{Spin}_{n}\right)=\operatorname{ed}\left(\operatorname{Spin}_{n}\right) \geq \operatorname{ed}\left(I_{n}^{3}\right) \geq \operatorname{ed}_{2}\left(I_{n}^{3}\right) .
$$

Hence, $\operatorname{ed}_{2}\left(I_{n}^{3}\right)=\operatorname{ed}\left(I_{n}^{3}\right)=\operatorname{ed}\left(\operatorname{Spin}_{n}\right)$. The latter value is known by Theorem 2.2.

Case $n \not \equiv 2(\bmod 4)$ and $n \geq 15$. Let $n=a+b$ with even $b \neq 2$. Let $Z$ be the trivial group if $b=0$ and the image of the center $C_{b}$ of $\operatorname{Spin}_{b}$ in $H_{a, b}$ if $b \geq 4$. Then $Z$ is central in $H_{a, b}$; hence, the group $H_{\text {ét }}^{1}(K, Z)$ acts on $H_{\text {êt }}^{1}\left(K, H_{a, b}\right)$.

Lemma 7.2. Let $\xi, \eta \in H_{e t}^{1}\left(K, H_{a, b}\right)$ with even $b \neq 2$. Suppose that $q(\xi)=q_{a} \perp q_{b}$ and $q(\eta)=q_{a} \perp \lambda q_{b}$ with the forms $q_{a} \in I_{a}^{2}(K)$ and $q_{b} \in I_{b}^{2}(K)$ and $\lambda \in K^{\times}$. Then $\eta=\alpha \xi$ for some $\alpha \in H_{e ́ t}^{1}(K, Z)$.

Proof. The statement is trivial if $b=0$, so assume that $b \geq 4$. The restriction of the natural homomorphism $H_{a, b} \rightarrow \mathbf{O}_{b}^{+}$to the subgroup $Z$ yields a surjection 
$\varphi: Z \rightarrow \boldsymbol{\mu}_{2}=\operatorname{Center}\left(\mathbf{O}_{b}^{+}\right)$. The kernel of $\varphi$ coincides with the kernel $C$ of the canonical homomorphism $H_{a, b} \rightarrow \mathbf{O}_{a}^{+} \times \mathbf{O}_{b}^{+}$.

As $Z$ is isomorphic to $\boldsymbol{\mu}_{2} \times \boldsymbol{\mu}_{2}$ or $\boldsymbol{\mu}_{4}$, the homomorphism $\varphi^{*}: H_{\text {ét }}^{1}(K, Z) \rightarrow$ $H_{\text {ét }}^{1}\left(K, \mu_{2}\right)=K^{\times} / K^{\times 2}$ is surjective. Let $\gamma \in H_{\text {ét }}^{1}(K, Z)$ be such that $\varphi^{*}(\gamma)=$ $\lambda K^{\times 2}$. Then $q(\gamma \xi)=q_{a} \perp \lambda q_{b}=q(\eta)$. Then there is $\beta \in H_{\text {ét }}^{1}(K, C)$ such that $\eta=\beta(\gamma \xi)$. Hence, $\eta=\alpha \xi$, where $\alpha=\beta^{\prime} \gamma$ with $\beta^{\prime}$ the image of $\beta$ under the map $H_{\text {êt }}^{1}(K, C) \rightarrow H_{\text {êt }}^{1}(K, Z)$ induced by the inclusion of $C$ into $Z$.

Let $\xi \in H_{\text {êt }}^{1}\left(K, \operatorname{Spin}_{n}\right)$ be such that the form $q=q(\xi) \in I_{n}^{3}(K)$ is generic for the functor $I_{n}^{3}$ (see [Merkurjev 2009, §2.2]). In particular, $\operatorname{ed}^{I_{n}^{n^{3}}}(q)=\operatorname{ed}\left(I_{n}^{3}\right)$. Note that $q$ is anisotropic.

Identifying $\boldsymbol{\mu}_{2}$ with the kernel of $\operatorname{Spin}_{n} \rightarrow \mathbf{O}_{n}^{+}$, we have an action of $H_{\text {ét }}^{1}\left(E, \boldsymbol{\mu}_{2}\right)=$ $E^{\times} / E^{\times 2}$ on $H_{\text {êt }}^{1}\left(E, \operatorname{Spin}_{n}\right)$, where $E=K((t))$. Consider the element $t \xi_{E} \in H_{\text {ét }}^{1}(E$, $\operatorname{Spin}_{n}$ ) over $E$. We claim that $t \xi_{E}$ is ramified. Suppose not, i.e., $t \xi_{E}$ comes from an element $\rho \in H_{\text {êt }}^{1}\left(O, \operatorname{Spin}_{n}\right)$, where $O=K \llbracket t \rrbracket$. Let $q^{\prime} \in H_{\text {êt }}^{1}\left(O, \mathbf{O}_{n}^{+}\right)$be the image of $\rho$ viewed as a quadratic form over $O$. We have

$$
q_{E}^{\prime}=q\left(t \xi_{E}\right)=q\left(\xi_{E}\right)=q_{E} ;
$$

hence, $q^{\prime}=q_{O}$. Then $\rho$ and $\xi_{O}$ belong to the same fiber of the map

$$
H_{\text {êt }}^{1}\left(O, \operatorname{Spin}_{n}\right) \rightarrow H_{\text {ét }}^{1}\left(O, \mathbf{O}_{n}^{+}\right) .
$$

As the group $H_{\text {et }}^{1}\left(O, \mu_{2}\right)=O^{\times} / O^{\times 2}$ acts transitively on the fiber, there is a unit $u \in O^{\times}$satisfying $t \xi_{E}=u \xi_{E}$. It follows from [Knus et al. 1998, Proposition 28.11] that $t u^{-1}$ is in the image spinor norm map

$$
\mathbf{O}^{+}\left(q_{E}\right) \rightarrow H_{\mathrm{ét}}^{1}\left(E, \boldsymbol{\mu}_{2}\right)=E^{\times} / E^{\times 2}
$$

for the form $q_{E}$; hence, $q$ is isotropic by [Elman et al. 2008, Theorem 18.3], a contradiction. The claim is proven.

Let $L / F$ be a subextension of $E / F$, and let $\eta \in H_{\text {êt }}^{1}\left(L, \operatorname{Spin}_{n}\right)$ be such that $\operatorname{tr} \cdot \operatorname{deg}(L / F)=\operatorname{ed}^{\operatorname{Spin}_{n}}(t \xi)$ and $\eta_{E} \simeq t \xi_{E}$. We have $q(\eta)_{E}=q(t \xi)=q\left(\xi_{E}\right)=q_{E}$; hence, the form $q(\eta)_{E}$ is anisotropic.

Let $v$ be the restriction on $L$ of the discrete valuation of $E$. As $t \xi$ is ramified, $v$ is nontrivial; hence, $v$ is a discrete valuation. Let $\pi \in L$ be a prime element.

Consider the completion $\hat{L}$ of $L$. As $E$ is complete, we can view $\hat{L}$ as a subfield of $E$. Write $q\left(\eta_{\hat{L}}\right)=\left(q_{a}\right)_{\hat{L}} \perp \pi\left(q_{b}\right)_{\hat{L}}$, where $q_{a}$ and $q_{b}$ are anisotropic quadratic forms over the residue field $\bar{L}$ of dimension $a$ and $b$, respectively. As $q(\eta) \in I^{3}(\hat{L})$, we have $q_{b} \in I^{2}(\bar{L})$, and therefore, $b$ is even and $b \neq 2$. By Lemma 4.3, there is $\eta^{\prime} \in H_{\text {ét }}^{1}\left(\hat{L}, H_{a, b}\right)$ that maps to $\eta$ with $q\left(\eta^{\prime}\right)=\left(\left(q_{a}\right)_{\hat{L}}, \pi\left(q_{b}\right)_{\hat{L}}\right)$.

We claim that the ramification index $e$ of the extension $E / \hat{L}$ is odd. Suppose $e$ is even. Note that $q_{a} \perp q_{b} \in I_{n}^{3}(\bar{L})$. Lemma 4.3 allows us to choose an unramified 
element $v \in H_{\text {ét }}^{1}\left(\hat{L}, H_{a, b}\right)$ with $q(v)=\left(\left(q_{a}\right)_{\hat{L}},\left(q_{b}\right)_{\hat{L}}\right)$. By Lemma 7.2, there is $\alpha \in H_{\text {êt }}^{1}(\hat{L}, Z)$ such that $\eta^{\prime}=\alpha \nu$. If $b$ is divisible by 4 , we have $Z \simeq \boldsymbol{\mu}_{2} \times \boldsymbol{\mu}_{2}$. As $e$ is even, $\alpha$ is unramified over $E$; hence, $\eta_{E}^{\prime}$ is unramified. It follows that $\eta_{E} \simeq t \xi$ is also unramified, a contradiction.

Suppose that $b \equiv 2(\bmod 4)$. Note that $0<b<n$ since $n \not \equiv 2(\bmod 4)$. Write $\pi=u t^{k}$ with a unit $u \in O^{\times}$and even $k$. Then

$$
\left(q_{a} \perp u q_{b}\right)_{E} \simeq\left(q_{a} \perp \pi q_{b}\right)_{E} \simeq q\left(\eta_{E}\right) \simeq q\left(t \xi_{E}\right)=q\left(\xi_{E}\right)=q_{E} .
$$

It follows that $q \simeq\left(q_{a}\right)_{K} \perp\left(\bar{u} q_{b}\right)_{K}$, i.e., $q$ contains the subform $\left(\bar{u} q_{b}\right)_{K}$ in $I^{2}(K)$ of dimension $b$. This contradicts Theorem 4.2. The claim is proven.

Thus, $e$ is odd. We have

$$
\left(q_{a} \perp u t q_{b}\right)_{E} \simeq\left(q_{a} \perp \pi q_{b}\right)_{E} \simeq q\left(\eta_{E}\right) \simeq q\left(t \xi_{E}\right)=q\left(\xi_{E}\right)=q_{E} .
$$

It follows that $\left(q_{b}\right)_{K}$ is hyperbolic and hence $\left(q_{a} \perp q_{b}\right)_{K}=\left(q_{a}\right)_{K}=q$ in $W(K)$, i.e., $\left(q_{a} \perp q_{b}\right)_{K} \simeq q$.

Note that $\left(q_{a}\right)_{\hat{L}}=\left(q_{a}\right)_{\hat{L}}+\pi\left(q_{b}\right)_{\hat{L}}=q\left(\eta_{\hat{L}}\right) \in I^{3}(\hat{L})$; hence, $q_{a} \in I^{3}(\bar{L})$ and $q_{a} \perp q_{b} \in I_{n}^{3}(\bar{L})$. Therefore, $q$ is defined over $\bar{L}$ for the functor $I_{n}^{3}$; hence,

$$
\text { ed }^{\operatorname{Spin}_{n}}(t \xi)=\operatorname{tr} \cdot \operatorname{deg}(L / F) \geq \operatorname{tr} \cdot \operatorname{deg}(\bar{L} / F)+1 \geq \operatorname{ed}^{I_{n}^{3}}(q)+1=\operatorname{ed}\left(I_{n}^{3}\right)+1 .
$$

It follows that ed $\left(\operatorname{Spin}_{n}\right) \geq \operatorname{ed}\left(I_{n}^{3}\right)+1$; hence, $\operatorname{ed}\left(I_{n}^{3}\right)=\operatorname{ed}\left(\mathbf{S p i n}_{n}\right)-1$ by Lemma 3.2. The value of ed $\left(\mathbf{S p i n}_{n}\right)$ is given in Theorem 2.2.

In what follows, we use the following observation (see [Berhuy and Favi 2003]): if a functor $\mathscr{F}$ admits a nontrivial cohomological invariant of degree $d$ with values in $\mathbb{Z} / 2 \mathbb{Z}$, then $\operatorname{ed}_{2}(\mathscr{F}) \geq d$.

Case $\boldsymbol{n}=7$. Every form $q$ in $I_{7}^{3}(K)$ is the pure subform of a 3 -fold Pfister form $\langle\langle a, b, c\rangle\rangle$; hence, $\operatorname{ed}\left(I_{7}^{3}\right) \leq 3$. On the other hand, the Arason invariant $e_{3}(q \perp\langle-1\rangle)=(a) \cup(b) \cup(c) \in H^{3}(K, \mathbb{Z} / 2 \mathbb{Z})$ is nontrivial (see [Garibaldi 2009, $\S 18.6])$; hence, $\operatorname{ed}_{2}\left(I_{7}^{3}\right) \geq 3$.

Case $\boldsymbol{n}=8$. Every form $q$ in $I_{8}^{3}(K)$ is a multiple $e\langle\langle a, b, c\rangle\rangle$ of a 3-fold Pfister form; hence, $\operatorname{ed}\left(I_{8}^{3}\right) \leq 4$. The invariant $a_{4}(q)=(e) \cup(a) \cup(b) \cup(c) \in H^{4}(K, \mathbb{Z} / 2 \mathbb{Z})$ is nontrivial; hence, $\operatorname{ed}_{2}\left(I_{8}^{3}\right) \geq 4$.

Case $n=9$ and 10. Every form $q$ in $I_{9}^{3}(K)$ or $I_{10}^{3}(K)$ is equal to $f \perp\langle 1\rangle$ or $f \perp\langle 1,-1\rangle$, respectively, where $f$ is a multiple of a 3 -fold Pfister form over $K$, by [Lam 2005, XII.2.8]. Hence, $I_{8}^{3} \simeq I_{9}^{3} \simeq I_{10}^{3}$.

Case $\boldsymbol{n}=11$. The degree-5 cohomological invariant $a_{5}$ of $\mathbf{S p i n}_{11}$ defined in [Garibaldi 2009, §20.8] factors through a nontrivial invariant of $I_{11}^{3}$; hence $\operatorname{ed}_{2}\left(I_{11}^{3}\right) \geq 5$. On the other hand, $\operatorname{ed}\left(I_{11}^{3}\right) \leq \operatorname{ed}\left(\operatorname{Spin}_{11}\right)=5$. 
Case $\boldsymbol{n}=12$. The degree- 6 cohomological invariant $a_{6}$ of $\mathbf{S p i n}_{12}$ defined in [Gari-

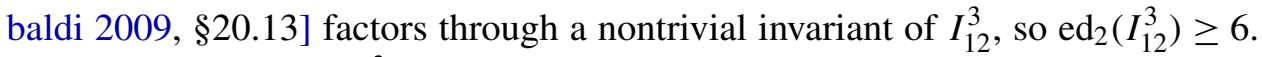
On the other hand, $\operatorname{ed}\left(I_{12}^{3}\right) \leq \operatorname{ed}\left(\operatorname{Spin}_{12}\right)=6$.

Case $n=13$ and 14. We know from the beginning of the proof (case $n \equiv 2(\bmod 4)$ and $n \geq 10)$ and from Theorem 2.2 that $\operatorname{ed}_{2}\left(I_{14}^{3}\right)=\operatorname{ed}\left(I_{14}^{3}\right)=\operatorname{ed}\left(\operatorname{Sppin}_{14}\right)=7$. By Lemma $4.5, \operatorname{ed}_{2}\left(I_{13}^{3}\right)=\operatorname{ed}_{2}\left(I_{13,0}^{3}\right) \geq \operatorname{ed}_{2}\left(I_{14,0}^{3}\right)-1=6$. On the other hand, $\operatorname{ed}\left(I_{13}^{3}\right) \leq \operatorname{ed}\left(\operatorname{Spin}_{13}\right)=6$.

\section{References}

[Berhuy and Favi 2003] G. Berhuy and G. Favi, "Essential dimension: a functorial point of view (after A. Merkurjev)”, Doc. Math. 8 (2003), 279-330. MR 2004m:11056 Zbl 1101.14324

[Brosnan et al. 2010] P. Brosnan, Z. Reichstein, and A. Vistoli, "Essential dimension, spinor groups, and quadratic forms", Ann. of Math. (2) 171:1 (2010), 533-544. MR 2011f:11053 Zbl 1252.11034

[Brosnan et al. 2011] P. Brosnan, Z. Reichstein, and A. Vistoli, "Essential dimension of moduli of curves and other algebraic stacks", J. Eur. Math. Soc. 13:4 (2011), 1079-1112. MR 2012g:14012 Zbl 1234.14003

[Èlašvili 1972] A. G. Èlašvili, "Stationary subalgebras of points of the common state for irreducible linear Lie groups", Funkts. Anal. Prilozh. 6:2 (1972), 65-78. In Russian; translated in Funct. Anal. Appl. 6:2 (1972), 139-148. MR 46 \#3690 Zbl 0252.22016

[Elman et al. 2008] R. Elman, N. Karpenko, and A. Merkurjev, The algebraic and geometric theory of quadratic forms, American Mathematical Society Colloquium Publications 56, American Mathematical Society, Providence, RI, 2008. MR 2009d:11062 Zbl 1165.11042

[Garibaldi 2009] S. Garibaldi, "Cohomological invariants: exceptional groups and spin groups", Mem. Amer. Math. Soc. 200:937 (2009). MR 2010g:20079 Zbl 1191.11009

[Knus et al. 1998] M.-A. Knus, A. Merkurjev, M. Rost, and J.-P. Tignol, The book of involutions, American Mathematical Society Colloquium Publications 44, American Mathematical Society, Providence, RI, 1998. MR 2000a:16031 Zbl 0955.16001

[Lam 2005] T. Y. Lam, Introduction to quadratic forms over fields, Graduate Studies in Mathematics 67, American Mathematical Society, Providence, RI, 2005. MR 2005h:11075 Zbl 1068.11023

[Lötscher 2013] R. Lötscher, "A fiber dimension theorem for essential and canonical dimension", Compos. Math. 149:1 (2013), 148-174. MR 3011881 Zbl 1260.14059

[Merkurjev 2009] A. S. Merkurjev, "Essential dimension”, pp. 299-325 in Quadratic forms - algebra, arithmetic, and geometry, edited by R. Baeza et al., Contemp. Math. 493, Amer. Math. Soc., Providence, RI, 2009. MR 2010i:14014 Zbl 1188.14006

[Popov 1987] A. M. Popov, "Finite isotropy subgroups in general position of irreducible semisimple linear Lie groups", Tr. Mosk. Mat. Obs. 50 (1987), 209-248. In Russian; translated in Trans. Mosc. Math. Soc. 1988 (1988), 205-249. MR 89a:20049 Zbl 0661.22009

[Reichstein 2000] Z. Reichstein, "On the notion of essential dimension for algebraic groups", Transform. Groups 5:3 (2000), 265-304. MR 2001j:20073 Zbl 0981.20033

[Reichstein and Youssin 2000] Z. Reichstein and B. Youssin, "Essential dimensions of algebraic groups and a resolution theorem for G-varieties", Canad. J. Math. 52:5 (2000), 1018-1056. MR 2001k:14088 Zbl 1044.14023 
[SGA 3 1970] M. Demazure and A. Grothendieck (editors), Schémas en groupes, III: Structure des schémas en groupes réductifs (Séminaire de Géométrie Algébrique du Bois Marie, 1962-1964), Lecture Notes in Mathematics 153, Springer, Berlin, 1970. MR 43 \#223c Zbl 0212.52810

[Vistoli 2005] A. Vistoli, "Grothendieck topologies, fibered categories and descent theory", pp. 1-104 in Fundamental algebraic geometry, Math. Surveys Monogr. 123, Amer. Math. Soc., Providence, RI, 2005. MR 2223406 Zbl 1085.14001

Communicated by Raman Parimala

Received 2013-03-27 Revised 2013-05-25 Accepted 2013-06-24

chernous@math.ualberta.ca Department of Mathematical Sciences, University of Alberta, Edmonton, AB T6G 2G1, Canada

merkurev@math.ucla.edu

Department of Mathematics, University of California, Los Angeles, Los Angeles, CA 90095-1555, United States 


\section{Algebra \& Number Theory}

msp.org/ant

\section{EDITORS}

MANAGING EDITOR

Bjorn Poonen

Massachusetts Institute of Technology

Cambridge, USA

\author{
EDITORIAL BOARD CHAIR \\ David Eisenbud \\ University of California \\ Berkeley, USA
}

\section{BOARD OF EDITORS}

Georgia Benkart

Dave Benson

Richard E. Borcherds

John H. Coates

J-L. Colliot-Thélène

Brian D. Conrad

Hélène Esnault

Hubert Flenner

Edward Frenkel

Andrew Granville

Joseph Gubeladze

Roger Heath-Brown

Ehud Hrushovski

Craig Huneke

Mikhail Kapranov

Yujiro Kawamata

János Kollár

Yuri Manin

Barry Mazur

Philippe Michel
University of Wisconsin, Madison, USA

University of Aberdeen, Scotland

University of California, Berkeley, USA

University of Cambridge, UK

CNRS, Université Paris-Sud, France

University of Michigan, USA

Freie Universität Berlin, Germany

Ruhr-Universität, Germany

University of California, Berkeley, USA

Université de Montréal, Canada

San Francisco State University, USA

Oxford University, UK

Hebrew University, Israel

University of Virginia, USA

Yale University, USA

University of Tokyo, Japan

Princeton University, USA

Northwestern University, USA

Harvard University, USA

École Polytechnique Fédérale de Lausanne
Susan Montgomery

Shigefumi Mori

Raman Parimala

Jonathan Pila

Victor Reiner

Karl Rubin

Peter Sarnak

Joseph H. Silverman

Michael Singer

Vasudevan Srinivas

J. Toby Stafford

Bernd Sturmfels

Richard Taylor

Ravi Vakil

Michel van den Bergh

Marie-France Vignéras

Kei-Ichi Watanabe

Efim Zelmanov

Shou-Wu Zhang
University of Southern California, USA

RIMS, Kyoto University, Japan

Emory University, USA

University of Oxford, UK

University of Minnesota, USA

University of California, Irvine, USA

Princeton University, USA

Brown University, USA

North Carolina State University, USA

Tata Inst. of Fund. Research, India

University of Michigan, USA

University of California, Berkeley, USA

Harvard University, USA

Stanford University, USA

Hasselt University, Belgium

Université Paris VII, France

Nihon University, Japan

University of California, San Diego, USA

Princeton University, USA

PRODUCTION

production@msp.org

Silvio Levy, Scientific Editor

See inside back cover or msp.org/ant for submission instructions.

The subscription price for 2014 is US $\$ 225 /$ year for the electronic version, and $\$ 400 /$ year $(+\$ 55$, if shipping outside the US) for print and electronic. Subscriptions, requests for back issues and changes of subscribers address should be sent to MSP.

Algebra \& Number Theory (ISSN 1944-7833 electronic, 1937-0652 printed) at Mathematical Sciences Publishers, 798 Evans Hall \#3840, c/o University of California, Berkeley, CA 94720-3840 is published continuously online. Periodical rate postage paid at Berkeley, CA 94704, and additional mailing offices.

ANT peer review and production are managed by EditFLOW ${ }^{\circledR}$ from Mathematical Sciences Publishers.

\section{PUBLISHED BY}

- mathematical sciences publishers

nonprofit scientific publishing

http://msp.org/

(C) 2014 Mathematical Sciences Publishers 


\section{Algebra \& Number Theory}

Volume $8 \quad$ No. $2 \quad 2014$

Large self-injective rings and the generating hypothesis

LEIGH SHEPPERSON and NEIL STRICKLAND

On lower ramification subgroups and canonical subgroups

303

SHIN HATTORI

Wild models of curves

DINO LORENZINI

Geometry of Wachspress surfaces

COREY IRVING and HAL SCHENCK

Daniel Goldstein, Robert M. GuRAlnick, Mark L. Lewis, AleXander Moretó, Gabriel Navarro and Pham HuU TieP

The homotopy category of injectives

AMNON NEEMAN

Essential dimension of spinor and Clifford groups

Vladimir Chernousov and AleXANDER MERKurJeV

On Deligne's category $\underline{\operatorname{Rep}}^{a b}\left(S_{d}\right)$

JONATHAN COMES and VICTOR OSTRIK

Algebraicity of the zeta function associated to a matrix over a free group algebra

CHRISTIAN KASSEL and Christophe REUTENAUER 